NOTA

\title{
Color de la madera de Acacia melanoxylon: aplicación del sistema CIE
}

\author{
Wood color of Acacia melanoxylon: CIE color system application
}

\author{
Silvia Monteoliva a*, Dora V Igartúa ${ }^{\mathrm{b}}$, Elías J Matta \\ *Autor de correspondencia: ${ }^{a}$ Universidad Nacional de La Plata, Facultad de Ciencias Agrarias y Forestales, CC 31 (1900), La \\ Plata, Argentina, tel.: 54-0221-4236616, fax: 54-0221-4252346, smonteoliva@yahoo.com.ar \\ b Universidad Nacional de Mar del Plata, Facultad de Ciencias Agrarias, Balcarce, Argentina. \\ c Universidad Nacional del Litoral, Facultad de Ingeniería Química, INTEC (UNL-CONICET), Santa Fe, Argentina.
}

\begin{abstract}
SUMMARY
The aims of this study were to describe wood color of Acacia melanoxylon with CIE color system and to analyze color variation between sapwood and heartwood among sites. Twenty trees were selected at four sites in Buenos Aires Province (Argentine). Destructive sampling was done, and one disc was taken at the base. Each disc was cut in radial boards $(2 \mathrm{~cm}$ thick) and four zones were demarcated: sapwood, two transitions zones (or external heartwood) and central heartwood. The parameters $\mathrm{x}$, y, Luminance, dominant wavelength (DWL) and purity excitation (EP) were measured. The $\mathrm{x}, \mathrm{y}$ and DW values differed among sites; although for luminance and EP no difference was found. Differences between sapwood and heartwood values were remarkable for all the variables. Sapwood and heartwood chromospheres were optically different. Sapwood color was light and varied between greenyellow zone and yellow saturated zone. Heartwood color predominates from yellow to saturated yellow orange. DWL ranges were 564-574 nm and low saturated (23-28\%). Dispersion variables inside heartwood showed no relationship between external and central heartwood sectoring; therefore, three zones with similar optical properties would be considered as heartwood.
\end{abstract}

Key words: CIE color system, color, sapwood, heartwood, Acacia melanoxylon.

\section{RESUMEN}

Los objetivos de este trabajo fueron describir el color de la madera de Acacia melanoxylon con el sistema colorimétrico CIE, y analizar la variabilidad entre la albura y el duramen y entre los sitios de crecimiento. Se seleccionaron 20 árboles en cuatro sitios del sudeste de la Provincia de Buenos Aires (Argentina). Se realizó un muestreo destructivo, tomando una rodaja (10-15 cm espesor) en la base. De cada rodaja se cortaron tablas radiales y se demarcaron cuatro sectores diferentes: albura, dos zonas de transición (duramen externo) y el centro del duramen. Se determinaron los parámetros de las coordenadas colorimétricas "x", "y", luminosidad, longitud de onda dominante (DWL) y pureza de excitación (EP). Los valores de " $x$ " e "y", y los de DWL variaron significativamente con el sitio, la luminosidad y la saturación del color, en cambio, no tuvieron diferencias con la localidad. Las diferencias entre los valores de duramen y albura fueron notables en todas las variables. Los cromóforos de la albura fueron ópticamente diferentes a los del duramen. Los colores de la albura resultaron claros variando de un verde-neutro-amarillento a un definido amarillo-parcialmente saturado. El duramen se extendió desde un bien definido amarillo parcialmente saturado al naranja más saturado. Las cotas DWL fueron 564-574 nm y la saturación fue baja (23-28\%). La dispersión de las variables dentro del duramen no estuvo relacionada con la sectorización en duramen externo y duramen central, por lo que podría considerarse a las zonas de transición con la albura y a la zona central del duramen como de similares propiedades ópticas.

Palabras clave: sistema CIE, color, albura, duramen, Acacia melanoxylon.

\section{INTRODUCCIÓN}

Desde hace más de un siglo la madera de Acacia melanoxylon $\mathrm{R}$. Br. es considerada internacionalmente como una de las maderas más decorativas del mundo, perteneciendo a la categoría del nogal, caoba y teca, y ha sido el puntal de la industria de muebles finos de Tasmania. Sus principales atributos tecnológicos están relacionados con el contenido de duramen y su color, características muy variables, que parecen estar influenciadas en mayor medida por el medio ambiente. En Australia se premia el precio según el color del duramen, que varía de marrón claro dorado a marrón oscuro pudiendo tener tintes rojizos y rayas (Nicholas y Brown 2002, Tasmanian Timber Promotion Board 2006). En algunos casos se ha expresado la preferencia por madera oscura y uniforme, en otros, el mercado ha requerido la presencia de rayas con otros pigmentos (Harrison 1975). 
Es dificultoso describir el color de la madera usando valores físicos ya que el mismo no es uniforme, y muestra figuras o vetas más o menos marcadas dependiendo del grano y textura de la madera (Nishino et al. 1998). Los métodos basados en la percepción humana del color no resultan consistentemente repetibles. Factores físicos y psicofísicos afectan la percepción visual de las personas acerca de las propiedades ópticas del material. Internacionalmente, el Comité de anatomistas de la madera, a través de su glosario de términos, definió cinco categorías subjetivas de color (IAWA Committee 1989). Los métodos colorimétricos son óptimos para obtener información objetiva sobre el color de la madera u otros materiales.

El sistema colorimétrico CIE ha sido utilizado para evaluar el color de tablas aserradas y de los nudos, determinar el deterioro de la madera, y detectar cambios de color con el secado o tratamientos térmicos (Phelps et al. 1994, Barry et al. 2003, Luostarinen y Mottonen 2004, Mononen et al. 2005). La caracterización colorimétrica de la madera de A. melanoxylon en el sistema CIE no tiene antecedentes. Harrison (1975) describió el color de la madera y los patrones de variación en el duramen para esta especie implantada en Sudáfrica. El autor evaluó subjetivamente el color a través de variables que denominó: oscuridad, uniformidad, y presencia y cantidad de pigmentos de color rojo, marrón, amarillo y gris. Las regiones de procedencia tuvieron efecto sobre la oscuridad, la uniformidad, la pigmentación marrón y gris, no así las localidades dentro de regiones.

Existen pocos estudios que midan las variaciones del color en especies de maderas valiosas ante características como los sitios, la edad, la posición en el fuste (Raymond y Bradley 2002). Por lo tanto, la aplicación de una metodología colorimétrica que genere información comparable contribuirá a ampliar la base de datos de color para redefinir usos en recursos forestales valorados en los mercados y no tan difundidos.

Los objetivos de este trabajo fueron describir el color de la madera de A. melanoxylon con el sistema colorimétrico CIE, y analizar la variabilidad entre la albura y el duramen y entre los sitios de crecimiento en la región del sudeste de Buenos Aires, Argentina.

\section{MÉTODOS}

El material estudiado correspondió a cuatro sitios de muestreo en la región sudeste de la Provincia de Buenos Aires (Argentina) en los que la especie se desarrolla con una estructura de masa potencialmente proveedora de material comercial. Se considera a la región homogénea climáticamente. Desde el punto de vista edáfico, el concepto central para calificar a los suelos de todos los sitios estudiados es el del "argiudol típico" (Culot 2000). Dos de los sitios representaron estaciones serranas que son aquellas donde parece estar más frecuentemente implantada en la región: Cinco Cerros (CC) (37 43' 59' S, 58 14' 30" O; 163 m s.n.m.) y Los Tuelches (LT) ( $37^{\circ} 55^{\prime} 67^{\prime \prime}$ S; $58^{\circ}$ 06' 27" O; 155 m s.n.m.) en el Partido de Balcarce. Los dos restantes representaron situaciones de llanura, con proximidad al mar: Las Cortaderas (LC) $\left(38^{\circ} 17^{\prime} 29^{\prime \prime} \mathrm{S}\right.$, $58^{\circ}$ 09’ 09" O; 31 m s.n.m.), Partido de Gral. Alvarado

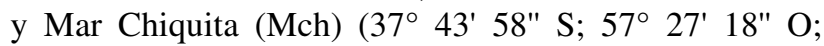
1 m s.n.m.), Partido de Mar Chiquita.

La selección de árboles se realizó, luego de un inventario, al azar y con dos restricciones: pertenecer a las clases diamétricas superiores y al estrato de los dominantes/ codominantes. Se seleccionaron 20 árboles de entre $12 \mathrm{~cm}$ y $33 \mathrm{~cm}$ de diámetro, cinco por sitio, y se procedió a su muestreo destructivo, tomando una rodaja en la base de 10 a $15 \mathrm{~cm}$ de espesor (0,3 $\mathrm{m}$ sobre nivel del suelo). De cada rodaja se cortaron tablas radiales de $2 \mathrm{~cm}$ de espesor, las cuales se cepillaron y pulieron en sus superficies radiales. Las edades, determinadas luego del muestreo, estuvieron comprendidas entre nueve y 32 años.

Inmediatamente antes de las mediciones colorimétricas, se demarcaron en las tablas cuatro sectores diferentes (posiciones radiales) señalados en la figura 1. Albura (S1), dos zonas de transición dentro del duramen (S2 y S4) consideradas duramen externo, y el centro del duramen (S3).

Mediciones colorimétricas. Las mediciones colorimétricas se realizaron con un equipo (espectrofotocolorímetro) ElrephoMat DFC5 (Carl Zeiss, Alemania), dotado de una esfera integradora recubierta con óxido de magnesio, iluminante D65 (lámpara de xenón emisión continua), geometría d $/ 10^{\circ}$. La apertura de medición fue de $20 \mathrm{~mm}$ de diámetro. La calibración se efectuó según normas con el patrón negro del equipamiento y sulfato de bario calibrado Merck. No se utilizaron filtros de corte, salvo mediciones esporádicas de verificación. Se mantuvo siempre activa la trampa de reflexión especular o trampa de brillo (normas CIE 1964).

La determinación de color requirió la medición de la reflexión producida por las muestras utilizando filtros a tres longitudes de onda (FMX 680 nm, FMY 557 nm y FMZ $450 \mathrm{~nm}$ ), retornando como resultado directo la reflexión difusa FMX, FMY, FMZ, y los valores triestímulo X, Y, Z. El cálculo permitió luego obtener las coordenadas de cromaticidad " $x$ " e " $y$ " para ingresar al espacio colorimétrico CIE. Para describir y analizar el color, se obtuvieron, además, la longitud de onda dominante (DWL, nm); la pureza de excitación (EP,\%) o saturación del color definido por una determinada DWL; y la luminosidad (Y,\%) (CIE 1994, TAPPI 1991, TAPPI 1994).

Se realizaron dos mediciones completas por cada sector de la probeta, las que luego se promediaron. Diversas muestras se midieron con/sin filtro de corte a $400 \mathrm{~nm}$ para verificar la posible presencia de inflorescencia UV.

Los resultados fueron analizados a través de recursos gráficos y estadísticos simples de posición y de dispersión. Se realizaron análisis de la varianza considerando al sitio y 


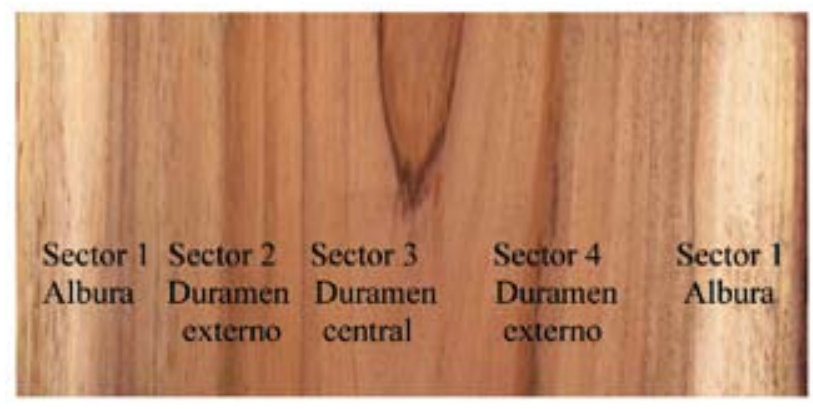

Figura 1. Sectores delimitados en la tabla. Sectors demarcated at board.

a las posiciones radiales o sectores (albura, duramen externo y duramen central) como factores fijos. Se complementaron los análisis con pruebas estadísticas de comparación de medias utilizando la prueba de Tukey.

\section{RESULTADOS}

La madera de A. melanoxylon presentó, a simple vista, una diferenciación de colores entre los diferentes sectores definidos en las tablas, y vetas de coloración más oscura que no coincidieron con la delimitación de los anillos de crecimiento (figura 2). Macroscópicamente, la descripción estándar según el glosario del Comité de anatomistas de la madera (IAWA Committee 1989) definió el color de esta madera como castaño o marrón amarillento a rojizo.

Desde la aplicación de la metodología CIE, esta madera presentó un comportamiento normal, sin evidencias de pigmentos fluorescentes al UV.

Los valores promedio generales de todos los árboles y sectores posicionaron al conjunto del material experimental según las coordenadas colorimétricas mostradas en él (cuadro 1). Se apreció un desplazamiento del color, mayor según el eje "x" y muy acotado según el eje "y". Las coordenadas " $x$ " e " $y$ ", y los valores de la longitud de onda dominante (DWL) variaron en un rango más acotado
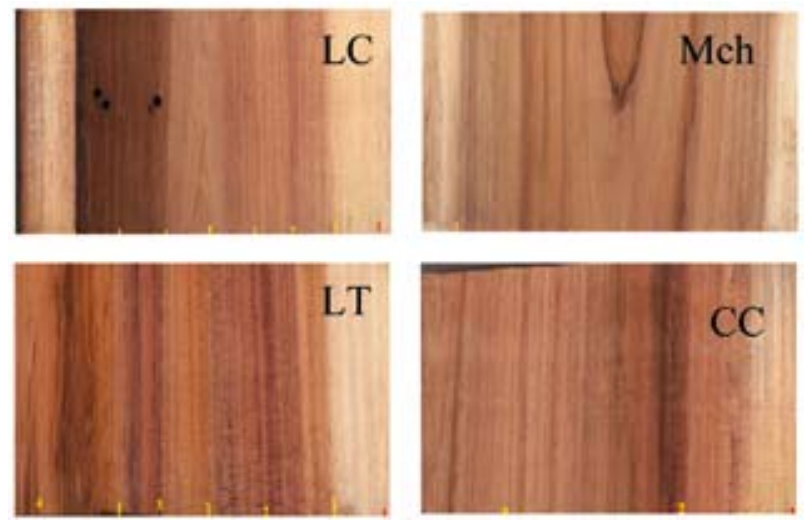

Figura 2. Tablas radiales de los cuatro sitios. LC: Las Cortaderas; Mch: Mar Chiquita; LT: Los Tuelches; CC: Cinco Cerros.

Radial boards from four sites.

en relación con la luminosidad y la saturación del color (EP) que presentaron una mayor dispersión (cuadro 1).

Los análisis de la varianza indicaron la ausencia de interacción significativa entre los factores sitio y sector. Los sitios fueron diferentes en su posicionamiento en cuanto a las coordenadas colorimétricas " $x$ " e " $y$ " como así también en la DWL, y no se diferenciaron en la saturación (EP) y luminosidad ("Y"). Las diferencias en las estadísticas colorimétricas de los cuatro sitios se expresan en el cuadro 1 (prueba de Tukey). El factor sector fue significativo. Cuando el ANDEVA se realizó sólo en el duramen, las diferencias significativas entre los sitios para DWL se mantienen. La luminosidad continúa no presentando diferencias, en cambio los valores de saturación del color (EP) resultaron significativamente distintos entre los sitios.

El comportamiento de todo el material (todas las muestras, todos los sectores) en el espacio-color desde las relaciones generales entre los valores de luminosidad ("Y") y " $\mathrm{x}$ ", e "y", mostró gráficamente la existencia de una relación inversa entre $Y$-" $x$ " en el rango de "x" $=0,32-0,40$ y de "Y" $=15,7-74,8 \%$ (figura 3). Asimismo, no se manifestó una relación clara entre "Y"-“y", con esta última variable

Cuadro 1. Estadística colorimétrica (media $\pm \mathrm{S}$ ) por sitio y promedio general. Prueba de Tukey.

Colorimetric statistics (average \pm SD) by sites and general average. Tukey's Test.

\begin{tabular}{lccccc}
\hline Variable** & Sitio CC & Sitio LC & Sitio LT & Sitio Mch & Promedio \\
\hline Edad & $20-31$ años & $19-32$ años & $17-30$ años & $9-15$ años & \\
"x" & $0,357 \mathrm{ab}^{*} \pm 0,016$ & $0,351 \mathrm{a} \pm 0,018$ & $0,364 \mathrm{~b} \pm 0,024$ & $0,352 \mathrm{a} \pm 0,018$ & $0,356 \pm 0,019$ \\
"y" & $0,388 \mathrm{a} \pm 0,003$ & $0,387 \mathrm{a} \pm 0,004$ & $0,388 \mathrm{a} \pm 0,006$ & $0,392 \mathrm{~b} \pm 0,007$ & $0,389 \pm 0,005$ \\
"Y" $(\%)$ & $41,3 \mathrm{a} \pm 12,5$ & $44,5 \mathrm{a} \pm 14,6$ & $39,3 \mathrm{a} \pm 15,7$ & $46,8 \mathrm{a} \pm 14,4$ & $43,13 \pm 14,32$ \\
DWL $(\mathrm{nm})$ & $567,7 \mathrm{bc} \pm 5,0$ & $565,3 \mathrm{ab} \pm 6,3$ & $568,5 \mathrm{c} \pm 8,8$ & $564,2 \mathrm{a} \pm 7,4$ & $566,3 \pm 6,99$ \\
EP $(\%)$ & $28,0 \mathrm{a} \pm 5,2$ & $25,8 \mathrm{a} \pm 6,0$ & $29,9 \mathrm{a} \pm 8,1$ & $27,7 \mathrm{a} \pm 6,8$ & $27,67 \pm 6,58$ \\
\hline
\end{tabular}

** "x": coordenada $\mathrm{x}$; "y": coordenada $\mathrm{y}$; "Y": luminosidad; DWL: longitud de onda dominante; EP: pureza de excitación

* Las letras se leen horizontalmente. Letras iguales no difieren significativamente $(P<0,05)$. 
acotada en el rango "y" = 0,38-0,40. La luminosidad decreció con el corrimiento hacia el naranja (mayores valores de "x"), lo que produjo el típico "marrón” (figura 3).

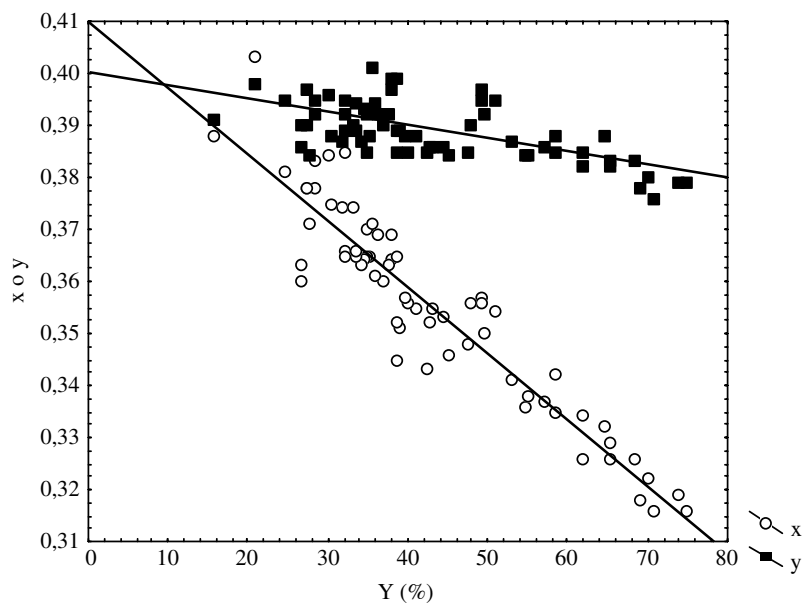

Figura 3. Relación entre las variables Luminosidad (Y) " $\mathrm{x}$ " e "y".

Relationship between Luminance (Y), " $\mathrm{x}$ " and " $y$ ".

El comportamiento general del conjunto del material fue un corrimiento desde un verde amarillento claro hacia el amarillo naranja mucho más oscuro.

Variación del color con la posición radial: albura y duramen. Las diferencias entre los valores de duramen (central S3 y duramen externo S2 y S4) y de la albura fueron significativas $(P=0,000)$ en todas las variables (cuadro 2).

La caracterización del duramen respecto a la de la albura indicó para el primero un corrimiento al rojo (mayores valores de "x"), una variación de $14 \mathrm{~nm}$ en DLW y una caída de casi $28 \%$ en Luminosidad. La estadística colorimétrica del duramen no difirió significativamente entre sus sectores externo y central (cuadro 2).

La figura 4 representa una imagen ampliada del diagrama cromático para todo el material experimental, del comportamiento de la albura y las tres zonas identificadas del duramen. La albura (S1) estuvo restringida a la zona gris verdosa más clara, en el rango " $\mathrm{x}$ " $=0,34-0,32$, "y" = 0,38-0,39. El duramen (sectores S2, S3 y S4) dominó todo el rango de " $x$ " restante, hasta el amarillo naranja. En la zona 4 se identifican un par de puntos extremos que podrían sugerir la presencia de madera anómala, posiblemente madera de tensión. Esto otorgó mayores desvíos estándares a su estadística colorimétrica (cuadro 2). Nuevamente se apreció el comportamiento similar de los sectores de transición o duramen externo y del duramen central para todas las variables. Desde el punto de vista colorimétrico el duramen (sectores de transición y duramen central) se extendió desde un bien definido amarillo bastante claro al naranja bien oscuro, no pudiendo adjudicarse a ninguno de sus sectores un espacio colorimétrico en particular dentro de este rango de variación (cuadro 2, figura 4).
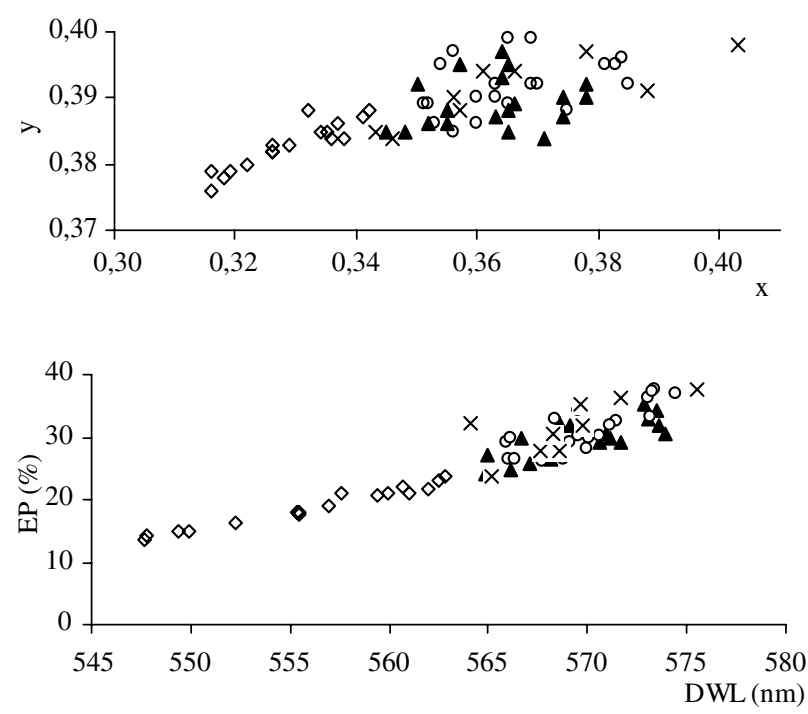
$\diamond$ Albura (S1)
$\Delta$ Duramen externo (S2)
- Duramen central (S3)
$\times$ Duramen externo $(\mathrm{S} 4)$

Figura 4. Albura, duramen externo $(\mathrm{S} 2, \mathrm{~S} 4)$ y duramen central (S3) representados en el espacio colorimétrico por sus coordenadas "x" e "y" y por su longitud de onda dominante (DWL) y pureza de excitación (EP).

Sapwood, external heartwood (S2, S4) and central heartwood (S3) represented at the colorimetric space by " $x$ ", and DWL and EP represented by "y".

Cuadro 2. Estadística colorimétrica (media $\pm \mathrm{S}$ ) discriminada por sectores radiales (albura- duramen externo-duramen central). Prueba de Tukey.

Colorimetric statistics (average \pm SD) according to radial zones (sapwood- external heartwood- central heartwood). Tukey's Test.

\begin{tabular}{lcccc}
\hline Variable** & Albura S1 & Duramen externo S2 & Duramen externo S4 & Duramen central S3 \\
\hline "x" & $0,329 \mathrm{a}{ }^{*} \pm 0,009$ & $0,363 \mathrm{~b} \pm 0,010$ & $0,367 \mathrm{~b} \pm 0,019$ & $0,366 \mathrm{~b} \pm 0,011$ \\
"y" & $0,383 \mathrm{a} \pm 0,004$ & $0,389 \mathrm{~b} \pm 0,004$ & $0,392 \mathrm{~b} \pm 0,006$ & $0,392 \mathrm{~b} \pm 0,004$ \\
"Y" $(\%)$ & $63,7 \mathrm{~b} \pm 6,9$ & $36,9 \mathrm{a} \pm 7,0$ & $34,4 \mathrm{a} \pm 10,4$ & $35,9 \mathrm{a} \pm 7,2$ \\
DWL (nm) & $556,3 \mathrm{a} \pm 5,2$ & $569,7 \mathrm{~b} \pm 3,0$ & $569,7 \mathrm{~b} \pm 4,0$ & $569,9 \mathrm{~b} \pm 2,7$ \\
EP $(\%)$ & $18,8 \mathrm{a} \pm 3,2$ & $29,6 \mathrm{~b} \pm 3,1$ & $31,7 \mathrm{~b} \pm 6,4$ & $31,3 \mathrm{~b} \pm 3,8$ \\
\hline
\end{tabular}

** "x": coordenada x; "y": coordenada y; "Y": luminosidad; DWL: longitud de onda dominante; EP: pureza de excitación

*Las letras se leen horizontalmente. Letras iguales no difieren significativamente $(P<0,05)$. 
El comportamiento, para todo el material, en términos de saturación del color (EP), indicó su aumento con el corrimiento hacia el naranja (figura 4), generando colores más "puros" o saturados, con menor influencia de los colores complementarios, particularmente el azul. Las variaciones de EP, de todos modos, no son muy importantes en términos absolutos, en especial en la albura (menos de 10 puntos). El duramen central (S3) y las zonas de transición (S2 y S4) estuvieron restringidos casi en su totalidad a un rango de EP que fue del 23 al 38\% y sólo una de las muestras superó el $40 \%$, mostrando que en general la saturación fue baja e importante la influencia del azul. Las cotas DWL fueron 564-574 nm (figura 4). No pudo adjudicarse a ninguno de los sectores definidos en el duramen, un espacio colorimétrico en particular dentro de este rango de variación en la saturación del color y en las longitudes de onda dominantes.

\section{DISCUSIÓN}

Bajo el modelo estadístico utilizado, el análisis detectó diferencias cromáticas en algunas de sus variables entre los materiales de los cuatro sitios estudiados dentro de la región sudeste de la Provincia de Buenos Aires que es considerada una unidad climo-edáfica homogénea (Culot 2000). Harrison (1975), en cambio, en su estudio para la especie en Sudáfrica, sólo encontró diferencias entre regiones y no entre localidades dentro de las mismas.

Cada tipo de cromóforo en la madera representa sólo un punto en el espacio-color y el diagrama cromático (con valores " $\mathrm{x}$ " e " $y$ " muy acotados). Los cromóforos usuales en la madera absorben mayoritariamente en el azul (440-460 $\mathrm{nm})$, y en menor medida en el verde $(560-570 \mathrm{~nm})$, por lo que el color resultante varía desde un amarillo verdoso al rojo amarillento. En el presente estudio los valores de " $\mathrm{x}$ " e "y" indicaron, para el material en su conjunto, la generación de diferentes cromóforos. La sectorización albura vs. duramen se apreció como la única fuente de variación en la estadística colorimétrica. Es posible que las diferencias en la edad de los individuos, los procesos oxidativos postcorte, la presencia de leño anómalo, la presencia de bandas oscuras, estén aportando a dicha variación, lo que deberá ser indagado en futuras investigaciones. Asimismo, serán necesarios estudios químicos adicionales para visualizar si los cromóforos son variantes del mismo compuesto o especies químicas diferentes.

La Luminosidad ("Y") de la madera se relaciona inversamente con la concentración de cromóforos. A mayor concentración, más oscura es la madera, situación representada por menores valores de luminosidad. Este comportamiento se observó claramente en los resultados, en donde la Luminosidad descendió $28 \%$ en el duramen con respecto a la albura.

La producción de cromóforos también aumenta con el proceso de duraminización. Desde el punto de vista fisioló- gico, la duraminización avanza con la edad y se establece un equilibrio con la cantidad óptima de albura que necesita el árbol. Es decir, que la formación del duramen actúa como una forma de regulación de la cantidad de albura (Taylor et al. 2002). Para varias especies las zonas de transición entre albura y duramen presentan diferencias en algunas propiedades (anatómicas, químicas y físicas) y por lo tanto puede esperarse que también presenten diferencias colorimétricas (Hillis 1987). En estudios anteriores sobre este mismo recurso regional de A. melanoxylon se encontraron diferencias en el patrón radial de otros atributos de la madera como la densidad seca al aire y la morfometría celular (Igartúa y Piter 2007, Monteoliva e Igartúa 2008, Igartúa et al. 2009). Sin embargo, en el presente no se registraron diferencias en las propiedades colorimétricas entre la transición o duramen externo y el duramen central. Por lo tanto, podrían considerarse a los tres sectores como duramen con similares propiedades ópticas.

\section{CONCLUSIONES}

La totalidad del material experimental del recurso regional de A. melanoxylon se ubicó en el espacio colorimétrico CIE desde el verde amarillento claro (albura) hasta el amarillo naranja mucho más oscuro (duramen), encontrándose diferencias en la DWL entre las localidades de procedencia.

Dentro del duramen no se registraron diferencias significativas según la sectorización en duramen externo y duramen central, por lo que podría considerarse a estas zonas como de similares propiedades ópticas.

Los cromóforos de la albura fueron ópticamente diferentes a los del duramen. Como consecuencia, los colores de la albura resultaron claros variando de un verdeneutro-amarillento a un definido amarillo-parcialmente saturado. El duramen se extendió desde un bien definido amarillo parcialmente saturado al naranja más saturado. Las cotas DWL fueron $564-574 \mathrm{~nm}$ y la saturación fue baja $(23-28 \%)$.

\section{REFERENCIAS}

Barry KM, NW Davies, CL Mohammend, L Beadle. 2003. Postharvest chemical staining in Blackwood (Acacia melanoxylon R.Br.). Holzforschung 57: 230-236.

Culot J.P. 2000. Caracterización edafo-climática de la región sudeste de la Provincia de Buenos Aires para Eucalyptus globulus. Primer seminario Internacional del Eucalyptus globulus en Argentina. Mar del Plata, Argentina. p. 2-9.

Commission Internattionale de L'Eclairage (CIE). 1994. A method of predicting corresponding colours under different chromatic and illuminance adaptations. CIE TR 109, Vienna.

Harrison CM. 1975. Heartwood colour patterns in South Africa Acacia melanoxylon. For. S. Afr. 17: 49-56.

Hillis WE. 1987. Heartwood and tree exudates. Berlin, Germany. Springer Verlag. 268 p. 
IAWA Committee. 1989. IAWA list of microscopic features for hardwood identification. IAWA Bull. 10(3): 219-332.

Igartúa DV, JC Piter. 2007. Propiedades físicas de la madera de Acacia melanoxylon R.Br. en el Sudeste de la Provincia de Buenos Aires-Argentina. III Congreso Iberoamericano de productos forestales (madereros y no madereros). IBEROMADERA 2007. Buenos Aires, Argentina. 8 p.

Igartúa DV, S Monteoliva, JC Piter. 2009. Estudio de algunas propiedades físicas de la madera de Acacia melanoxylon en Argentina. Revista Madera, Ciencia y Tecnología 11(1): 3-18.

Luostarinen K, V Mottonen. 2004. Effects of log storage and drying on birch (Betula pendula) wood proanthocyanidn concentration and discoloration. J. Wood Sci 50: 151-156.

Mononen K, L Alvila, T Pakkanen. 2005. Changes in color and structure of birch wood (Betula pendula) caused by bleaching with hydrogen peroxide solution. Holzforschung 59: 59-64.

Monteoliva S, DV Igartúa. 2008. Morfología fibrosa, composición química y densidad de Acacia melanoxylon. Estudios preliminares sobre el recurso en Argentina. V Congreso Iberoamericano de Celulosa y Papel (CIADICYP), México, octubre 2008. $10 \mathrm{p}$.
Nicholas I, I Brown. 2002. Blackwood-A Handbook for Growers and End Users. Forest Research Bulletin (Rotorua, N.Z.), No. 225.95 p.

Nishino Y, G Janin, B Chanson, P Detienne, J Gril, B Thibaut. 1998. Colorimetry of wood specimens from French Guiana. J. Wood Sci. 44: 3-8.

Phelps JE, DD Stokke, AD Pugel. 1994. Color analysis of white oak, edge-glued furniture panel stock. Forest Product J. 44(2): 35-38.

Raymond CA, AD Bradley. 2002. Assessment of wood colour in Eucalyptus nitens. Appita Journal 55 (2): 118-122.

TAPPI (Technical Association of Pulp and Paper Industry, USA). 1991. Optical measurement terminology. TIS 0804-02.

TAPPI (Technical Association of Pulp and Paper Industry, USA). 1994. Color of paper and paperboard (d/0 $0^{\circ}$ geometry). T 527 om-94.

Tasmanian Timber Promotion Board. 2006. Blackwood: Acacia melanoxylon. Consultado 10 de oct. 2007. Disponible en: http://www.tastimber.tas.gov.au/species/pdfs/blackwood. pdf.

Taylor AM, BL Gartner, JJ Morrell. 2002. Heartwood formation and natural durability. A review. Wood and Fiber Science 34(4): 587-611. 